\title{
ENSEIGNEMENT SCIENTIFIQUE ET LIGNES DE PARTAGE DISCIPLINAIRE
}

\author{
LA PLACE DE LA PHYSIQUE \\ DANS LA PREMIËRE MOITIE DU XIX ${ }^{e}$ SIÈCLE \\ Nicole HuLIN
}

Résume: L'organisation de l'enseignement scientifique s'effectue en suivant un découpage disciplinaire et la spécialisation des études impose des choix de couplage entre disciplines. Ainsi, apparaît un souci de classification dans la définition du domaine de chacune des disciplines de même que dans l'explicitation de leurs dépendances mutuelles. En France, la physique occupe une place charnière, éclatée en une physique expérimentale liée à la chimie et une physique mathématique rattachée aux sciences mathématiques; quant aux mathématiques, elles sont considérées comme un instrument nécessaire dans l'acquisition des connaissances scientifiques.

L'étude de l'enseignement scientifique nécessite une analyse suivant les différentes composantes disciplinaires (mathématiques, physique, chimie, histoire naturelle) qui ont chacune leur spécificité. Pour l'organisation des cursus, des découpages disciplinaires sont effectués et certains couplages retenus; il importe donc de préciser l'articulation des diverses disciplines scientifiques entre elles et de noter les lignes de partage.

Nous envisagerons l'organisation de l'enseignement scientifique, depuis l'établissement de l'Université impériale, en le stratifiant en trois niveaux : le haut enseignement dispensé dans les facultés, les études à l'École normale et l'agrégation des lycées, les études secondaires. Mais avant d'aborder l'organisation institutionnelle dans la première moitié du $\mathrm{XIX}^{\mathrm{e}}$ siècle, nous noterons la préoccupation d'expliciter les dépendances mutuelles des disciplines, de définir chacune des disciplines ou même sous-disciplines, de préciser les limites de leur domaine, à travers un certain nombre de textes (introductions de cours, rapports) qui, depuis le milieu du xviII ${ }^{e}$ siècle $^{1}$, font apparaître un souci de classification.

1. Pour l'enseignement des sciences au xvıI' siècle, voir l'article de François de DainvileE, "L'enseignement scientifique dans les collèges de Jésuites ", in L'Enseignement et la diffusion des sciences au xvif siècle, éd. René Taton, Paris, Hermann, 1986, p. 35-60, ou encore l'article de Lawrence W. B. BRockLIss, in Histoire des universités en France, dir. Jacques VERGER, Toulouse, Privat ("Bibliothèque historique Privat»), 1986, p. 216-222.

Revue de synthèse : IV S. $\mathrm{N}^{\mathrm{os}} 1-2$, janv.-juin 1994. 
Dans les préfaces ou introductions de cours de physique est affirmée la nécessité du recours aux mathématiques dont la maîtrise est jugée désormais essentielle pour l'étude de la physique. Ainsi, dans son Cours de physique expérimentale, Jean Théophile Désaguliers ${ }^{2}$ affirme qu'il faut faire appel à la géométrie et à l'arithmétique si on ne veut pas se borner à l'histoire naturelle et à la physique conjecturale ; cette nécessité est d'ailleurs soulignée avec beaucoup de force en faisant référence à Newton :

"C'est au chevalier Newton, qui le premier appliqua la Géométrie à la Philosophie, que nous sommes redevables de la déroute de cette armée de Goths et de Vandales, qui ravageaient le monde philosophique. »

Mais J.T. Désaguliers note aussi la différence entre la physique et les mathématiques (les mathématiques pures commençant toujours par la définition des termes, ce qui élimine toute possibilité de dispute) et il définit « les mathématiques mixtes où l'on raisonne mathématiquement sur des objets physiques ".

Cette affirmation du lien mathématiques-physique se retrouve dans le Cours de physique expérimentale et mathématique de Pierre Van Mussenbrock $^{3}$. Notant les connaissances dont dépendent les progrès de la physique, il précise le rôle de la connaissance mathématique :

"C'est par elle qu'on observe l'intensité des causes, toute l'étendue des propriétés et des phénomènes, et qu'on détermine ce qui doit suivre de ces découvertes. »

Mais, préalablement, il a défini la physique qui « a pour objet le corps, l'espace et le mouvement ", ainsi que le phénomène c'est-à-dire " tout ce que nous découvrons à l'aide des sens ". Cependant, ici, la physique est aussi présentée par son utilité : elle est d'un « secours sans bornes pour toutes les commodités de la vie ", elle "empêche de donner dans toutes les superstitions ", elle " fournit des preuves incontestables de l'existence de Dieu ". Ces affirmations sont à rapprocher de celles de Georges Cuvier (1807) ou de l'économiste saint-simonien Michel Chevalier (1846)

2. Jean Théophile Desaguliers, Cours de physique expérimentale, trad., Paris, J. Rollin et Ch. A. Jombert, 1751, t. I, p. ii, iv, 1, 2.

3. Pierte VAN MUSSENBROCK, Cours de physique expérimentale et mathématique, trad. par Joseph Sigaud de La Fond, Paris, Ganeau, 1769, t. I, p. 6, 15. 
lorsqu'ils veulent convaincre de l'utilité et de la valeur de l'étude des sciences ${ }^{4}$ pour en obtenir le développement.

$\mathrm{Si}$, dans ses Leçons de physique expérimentale, l'abbé Nollet ${ }^{5}$ note aussi " comme une chose nécessaire à celui qui veut étendre les progrès de la physique, de posséder assez la géométrie et l'algèbre pour s'en aider dans ses recherches, et pour évaluer ses découvertes ", il a préalablement situé la physique expérimentale, qui «entreprend de nous dévoiler le méchanisme de la nature ", par rapport à l'histoire naturelle qui « offre [...] l'inventaire de nos richesses ". Cet auteur éprouve donc non seulement le besoin d'affirmer la nécessité des mathématiques pour la physique mais il manifeste aussi le souci de préciser le domaine de la physique par rapport à l'histoire naturelle en opposant la connaissance du " méchanisme" à " l'inventaire ".

C'est au sein de la triade physique-chimie-histoire naturelle que le chimiste Fourcroy ${ }^{6}$ situe la chimie. Il la définit comme une science physique ou naturelle, précise en quoi elle diffère, d'une part, de l'histoire naturelle " qui décrit les corps dans leur intégrité " et, d'autre part, de la physique " qui ne s'occupe que des corps étendus, soumis à la mesure, calculables dans leur masse, leur surface, leur densité, etc., et qui détermine l'action que les masses exercent les unes sur les autres ". Son souci essentiel, en fait, est de situer la chimie par rapport à la physique : « les molécules invisibles sont les vrais sujets chimiques, dit-il, tandis qu'on nomme sujets physiques tous les corps visibles dont on peut déterminer les dimensions et étudier les masses. " Il précise d'ailleurs la limite entre les deux en utilisant la notion de destruction (déjà présente dans la distinction chimie-histoire naturelle) :

“C'est parce qu'on détruit les masses pour traiter ou étudier chimiquement les corps qu'on a dit que le chimiste commençait où finissait le physicien. "

Dans cet examen de la définition des lignes de partage disciplinaire présentée dans les cours, les leçons à l'École normale de l'an III ${ }^{7}$ présentent un intérêt tout particulier. En effet, les enseignements dans les

4. Cf. Nicole Hulin-Jung, L'Organisation de l'enseignement des sciences, Paris, Comité des travaux historiques et scientifiques, 1989 , p. 47, 127-128.

5. Abbé Nollet, Leçons de physique expérimentale, $6^{\circ}$ éd., Paris, Durand, Neveu, 1771, préf., p. LXXXIX-XL et XLVII.

6. Antoine François de Fourcroy, Philosophie chimique, $1^{\text {re }}$ éd. $1792,3^{\circ}$ éd. Paris, Tourneisen, 1806, introd., p. 6-10.

7. Les Écoles normales, Paris, Imprimerie du Cercle social, 1800. 
diverses disciplines interviennent sur un pied d'égalité, sont dispensés dans un même contexte et par des savants de même rang. On constate que le souci de définir le domaine enseigné croît des mathématiques à la physique, la chimie et l'histoire naturelle, et indépendamment de l'ordre dans lequel les premières leçons ont lieu. Dans leur programme ou leur première leçon, les différents savants chargés des cours définissent le domaine de l'enseignement dont ils sont chargés en le situant par rapport à d'autres disciplines.

Dans la présentation de leur programme en mathématiques, Lagrange et Laplace ${ }^{8}$ sont extrêmement rapides sur la question, soulignant simplement l'enrichissement réciproque des mathématiques et de la physique. En effet, le domaine des mathématiques s'est accru par leur « application aux phénomènes de la nature, phénomènes qui sont les résultats mathématiques d'un petit nombre de lois invariables ", mais cette application « a ouvert de nouvelles routes dans l'analyse : c'est ainsi que les sciences, par leurs rapprochements, se prêtent de mutuels secours ».

Haüy $^{9}$, dès le début de l'exposé de son programme, définit l'objet de la physique comme «la connaissance des phénomènes de la nature et des lois dont ils dépendent ". Mais, compte tenu de ses centres d'intérêt personnels ${ }^{10}$, il situe la physique par rapport à l'histoire naturelle, opposant " théorie » et " classification » :

« Le naturaliste cherche dans l'aspect des individus, des points de comparaison propres à établir leur classification; le physicien, par le rapprochement des faits observés, parvient à les lier ensemble dans une même théorie. »

Cependant, exposant le plan de son cours, il en indique les limites par rapport au domaine de la chimie, d'une part, et des sciences physicomathématiques, d'autre part, et fait ainsi apparaître le rôle charnière de la physique située entre la chimie et les mathématiques. Toutefois, Haüy est beaucoup plus explicite sur la définition des domaines de la physique et de la chimie dans le Traité élémentaire de physique ${ }^{11}$, rédigé à l'intention des lycées et qui a pour base essentielle ses leçons à l'École normale de l'an III. Ainsi, on est dans le domaine de la physique si l'on considère "dans les corps les propriétés générales et permanentes" (ou si les changements subis sont passagers) et si « les lois qui déterminent les actions réciproques des mêmes corps se propagent à des distances plus ou moins considérables " ; par contre, on est dans le domaine de la chimie

8. Ibid., t. I, p. 16 .

9. Ibid., p. 32-33.

10. Après s'être intéressé à la botanique, Haüy était venu à l'étude de la minéralogie.

11. Abbé René Just Haư, Traité élémentaire de physique, Paris, Courcier, 1806, p. i-iij. 
«lorsque les phénomènes dépendent d'une action intime que les molécules des corps exercent les unes sur les autres, à des distances presque infiniment petites ». Mais Haüy ressent le besoin de présenter le troisième élément de la triade, l'histoire naturelle définie par son but de « classer » et de " décrire ", et qui comprend elle-même trois sciences, la zoologie, la botanique et la minéralogie. Immédiatement après avoir fait ces distinctions, il nuance son propos en affirmant l'existence d' " une seule et même science" subdivisée pour que «les différents esprits pu[i]ssent partager entre eux l'étude des diverses branches "; ainsi " plusieurs sciences se rencontrent auprès d'une même vérité ", tel est le cas de la théorie de la chaleur qui est à la limite du domaine du physicien et du chimiste. Nos connaissances "remontent toutes à une même unité " et les distinctions faites proviennent uniquement "des bornes de notre esprit et de celles du temps qui nous est accordé pour les cultiver ». Haüy définit donc les domaines de chaque discipline, mais signale des points de recouvrement, et affirme l'unité de la science.

Berthollet ${ }^{12}$, dans son programme, définit la chimie au sein de la physique dont l'étude "embrasse tous les phénomènes de la nature", ceux-ci devant être envisagés sous différents points de vue. Il distingue ainsi d'abord la physique générale et la physique particulière; reste alors la «classe de phénomènes qui sont dus à l'action interne des molécules des corps " : si l'action dépend de "l'organisation " c'est le domaine de la physique animale ou végétale, si elle en est indépendante c'est le domaine de la chimie. On retrouve donc ici le souci du chimiste de situer sa discipline au sein de la triade physique proprement dite-chimiehistoire naturelle.

Dans sa première leçon, le naturaliste Daubenton ${ }^{13}$ est de loin celui qui développe le plus longuement la définition de son domaine d'étude. Après avoir défini l'histoire naturelle qui « comprend les observations qui ont été faites sur les productions de la nature », il en précise les limites avec la chimie et la métallurgie. Il insiste fortement sur les différences entre l'histoire naturelle et la chimie, et indique que la "destruction » ${ }^{14}$ est le "point de partage " :

12. Claude Louis Berthollet, in op. cit. supra n. 7, t. I, p. 205-206.

13. Louis Daubenton, in op. cit. supra n. 7, t. I, p. 96-99.

14. Cette affirmation est à rapprocher de celle de R.J. HAơv, in Traité de cristallographie, Paris, Bachelier, 1822, p. XI, XIV-XV, quand il explique à propos de sa classification fondée sur la notion d'espèce en minéralogie (une controverse s'était établie avec le chimiste Berthollet) : “c'est à la cristallographie plutôt qu'à la chimie qu'appartient la distinction des espèces [...] la condition essentielle, qui exige que l'espèce soit représentée, ne peut être remplie que par celle des deux sciences qui nous dépeint les minéraux tels que les a produits la nature, et non celle qui ne nous les fait connaitre qu'à l'aide d'une opération dont les résultats ont effacé leurs traits caractéristiques. " 
«L'objet de l'histoire naturelle est fort différent de celui de la chimie, dans l'état présent de ces deux sciences. Le naturaliste observe les productions de la nature, telles qu'il les aperçoit [...] il ne se propose pas de les décomposer [...] ni de les détruire pour en rechercher les élémens. "

«[La] destruction est le point de partage entre l'étude du naturaliste et celle du chimiste. "

Et il ajoute plus loin qu'on distinguera toujours la chimie de l'histoire naturelle « comme l'on distingue les produits de l'art des productions de la nature ». Ayant ainsi réglé le problème des différences de la chimie et de l'histoire naturelle, il en vient à préciser le contenu de cette dernière ; par là, il est amené à distinguer l'histoire naturelle de l'anatomie, base de la physiologie et par conséquent de la médecine, par référence à nouveau à la notion de respect de l'intégrité :

« Le naturaliste doit visiter les parties intérieures du corps des animaux [...] sans détruire les formes, et sans rendre les objets méconnaissables par toutes les dissections nécessaires pour l'anatomie. »

De l'ensemble de ces considérations, il tire des conclusions à propos de l'enseignement pour lequel la spécialisation est nécessaire; il faut bien connaître les limites des sciences qu'on professe, " afin d'éviter toute équivoque, et de mettre la plus grande précision dans l'enseignement » à transmettre.

" [...] il y a une différence entre l'avancement des sciences en général et l'étude d'une science en particulier, et l'enseignement que l'on en donne. Lorsqu'on embrasse plusieurs sciences à la fois, c'est une curiosité indiscrète ; une sorte de libertinage d'esprit, qui fait qu'on ne les approfondit pas, et que l'on ne peut en donner de bonnes instructions. "

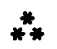

Dans ces présentations de cours apparaît nettement le souci de définir et de délimiter le domaine enseigné tout en précisant les articulations avec les disciplines connexes. Comme cela sera clair dans les Rapports $\grave{a}$ l'Empereur sur les progrès des sciences (1808) rédigés par Delambre et Cuvier, la physique occupe une place charnière au sein des sciences, prise en étau entre les mathématiques et la chimie. En effet, dans le rapport de Cuvier, concernant les sciences naturelles, la physique se trouve placée dans la partie consacrée à la chimie et dans la première des trois rubriques : chimie générale, chimie proprement dite, chimie particulière ; 
cependant, ce n'est pas toute la physique, la physique mathématique (c'est-à-dire la partie de la physique qui peut être soumise au calcul) est intégrée dans le rapport de Delambre ${ }^{15}$ sur les sciences mathématiques. D'ailleurs, celui-ci explique :

" À mesure que les sciences font des progrès et que leurs limites s'étendent, on voit diminuer l'espace qui les séparait, et la ligne de démarcation devient plus difficile à tracer. ")

Le souci de présenter et de classer les différentes disciplines se trouve encore, par exemple, dans la table analytique établie par François S. Beudant ${ }^{16}$, mais avec une mention spéciale des « liaisons des sciences mathématiques et des sciences physiques » et en précisant le but de la physique ainsi que celui de la chimie. On notera, au milieu du $\mathrm{xIx}^{\mathrm{e}}$ siècle, le maintien du soin de définir l'objet de la physique au début des manuels, mais d'une manière très synthétique. Ainsi, dans l'ouvrage d'A. Ganot ${ }^{17}$ de 1854 , la physique est présentée comme « l'étude des phénomènes terrestres " (élimination donc du domaine de l'astronomie) « inorganiques » (définition de la limite par rapport à l'histoire naturelle), " sans modification de leur substance » (différence avec la chimie).

LA PHYSIQUE DANS L'ENSEIGNEMENT

\section{Le haut enseignement dispensé dans les facultés}

Après la fondation de l'Université impériale, des chaires sont créées en 1809 , regroupées, dans l'ordre, suivant les trois rubriques: sciences mathématiques, sciences physiques et sciences naturelles. En sciences mathématiques, les trois chaires concernent le calcul différentiel et intégral, la mécanique, l'astronomie physique avec respectivement pour titulaire Sylvestre François Lacroix, Siméon Denis Poisson et Jean-Baptiste

15. Jean-Baptiste Delambre, in Rapports à l'Empereur sur les progrès des sciences, des lettres et des arts depuis 1789. T. I : Sciences mathématiques, Paris, Belin, 1989, p. 220.

16. François S. BEUdANT, Essai d'un cours élémentaire et général de sciences physiques, Paris, Tilliard, Croullebois, Verdière, 1815.

17. A. Ganot, Traité élémentaire de physique expérimentale et appliquée, Paris, chez l'Auteur, 1854, p. 1 et 2. 
Biot; ces chaires se diversifieront au cours de la première moitié du $\mathrm{XIX}^{e}$ siècle avec l'adjonction d'une chaire d'algèbre supérieur, puis d'une chaire de physique mathématique et calcul des probabilités, de mécanique physique et expérimentale, d'astronomie mathématique et mécanique céleste. À cette diversification progressive des chaires de sciences mathématiques s'oppose une remarquable stabilité des autres chaires avec, en sciences physiques, une chaire de physique theorique et expérimentale, une chaire de chimie et une de minéralogie (dont les premiers titulaires sont respectivement Gay-Lussac, Thenard et Haüy) et, en sciences naturelles, une chaire de zoologie et physiologie et une chaire de physique végétale. On remarquera la place de la chaire de minéralogie au sein des sciences physiques; d'abord occupée par R.J. Haüy, elle sera confiée ensuite à F.S. Beudant (1822), tous deux auteurs de traité de physique. Il convient aussi de noter le passage de Pierre-Louis Dulong dans la chaire de chimie (1820), comme adjoint de Louis-Jacques Thenard, avant sa nomination (1832) dans la chaire de physique. Entre 1809 et 1849, six professeurs se succèdent au poste de doyen; parmi eux, aucun titulaire de la chaire de physique : trois pour les sciences mathématiques (1809 S. F. Lacroix, 1840 S. D. Poisson, puis J.-B. Biot), deux pour la chimie (1821 L.-J. Thenard, 1842 J. B. Dumas) et un pour les sciences naturelles (1849 Henri Milne Edwards).

L'organisation des cours à la faculté des Sciences de Paris (statut du 16 février 1810) montre encore la place charnière de la physique entre les sciences mathématiques et la chimie, ainsi que l'étroit couplage entre la chimie et l'histoire naturelle. Deux séries de cours sont prévues : la série mathématique (calcul différentiel et intégral, mécanique, astronomie) et la série physique (chimie, histoire naturelle) avec en commun un cours de physique générale et expérimentale.

En ce qui concerne les grades, la question essentielle est celle de la spécialisation. Si la suprématie des mathématiques est consacrée par le décret de 1808 qui impose, pour être licencié des facultés des sciences, de répondre sur la statique et le calcul intégral, dès 1810 (statut du 16 février) la spécialisation apparaît : questions de calcul différentiel et intégral, mécanique, ou questions de physique et chimie, ou questions d'histoire naturelle suivant la destination des candidats, mention en étant faite dans les diplômes. La distinction explicite des trois licences apparaît dans le règlement du 8 juin 1848 ; alors, la minéralogie trouve place aux côtés de la chimie dans la licence ès sciences physiques et des épreuves pratiques sont introduites dans les licences concernant les sciences expérimentales. 
Quant au doctorat ${ }^{18}$ il est, dès 1808 , spécialisé avec deux thèses à soutenir soit sur la mécanique et l'astronomie, soit sur la physique et la chimie, soit sur les trois parties de l'histoire naturelle. Ainsi, tant dans la licence que dans le doctorat, physique et chimie sont couplées; toutefois, en 1848, une possibilité de spécialisation est introduite avec la tolérance d'une thèse unique. Mais il faut noter que les travaux de physique mathématique conduisent au $\mathrm{XIX}^{\mathrm{e}}$ siècle à des thèses de sciences mathématiques, avec par conséquent un éclatement de la physique puisque les travaux de physique expérimentale conduisent à des thèses de sciences physiques. Entre 1811 et 1850 , sur 50 thèses de sciences mathématiques à Paris, $22 \%$ concernent la physique mathématique ( $26 \%$ de 1811 à 1837 et $19 \%$ de 1838 à 1850). En ajoutant les thèses soutenues en province, les résultats ne sont pas fondamentalement changés, les pourcentages sont légèrement abaissés : pour 80 thèses, $20 \%$ de 1811 à 1837 et $15 \%$ de 1838 à 1850. Mais il convient aussi de prendre en compte la proportion de thèses de physique mathématique et de physique expérimentale : ces dernières sont 4 (Paris seulement) à 5 (Paris et province) fois plus nombreuses - dans la deuxième moitié du xIx $^{e}$ siècle, la proportion deviendra beaucoup plus écrasante.

Il importe aussi pour notre propos de dire quelques mots du concours d'agrégation des facultés des sciences (création du 28 mars 1840, règlement du 7 avril $1840^{19}$ ). Par la place qui y est faite à la physique mathématique, au sein du concours pour les sciences mathématiques, on sent toute l'influence de Poisson; en effet, la première des trois épreuves, la composition écrite, porte sur un sujet pris dans la mécanique appliquée à la physique ou à l'astronomie, et dans « toutes les questions importantes de physique ${ }^{20}$ qui peuvent donner lieu à des développements d'analyse transcendante " et la deuxième épreuve, l'argumentation, porte sur les mathématiques pures et la physique mathématique. Dans le rapport présenté au roi sur l'institution des agrégés de facultés, Victor Cousin justifiait l'exigence des trois licences pour chacun des trois ordres d'agrégation en insistant sur les connexions disciplinaires, illustrées par des exemples :

«Que deviendrait la physique mathématique, cette gloire de la France, si de sérieuses études de physique expérimentale ne se rencontraient dans le même esprit avec le talent de l'analyse? Sans une suffisante instruction en mathématiques, la cristallographie n'eût jamais été créée [...] ${ }^{21}$.

18. Nicole Hulin, "Les doctorats dans les disciplines scientifiques au xix ${ }^{e}$ siècle ", Revue d'histoire des sciences, XLIII/4, 1990, p. 401-426.

19. N. Hulin-Jung, op. cit. supra n. 4, p. 223-240.

20. Souligné par nous.

21. Victor Cousin, Euvres, Instruction publique, Paris, Pagnerre, 1850, t. I, p. 283. 


\section{Les études à l'École normale et l'agrégation des lycées}

Au début du $\mathrm{xIx}^{\mathrm{e}}$ siècle, la spécialisation n'existe ni dans les études de la section des sciences de l'École normale ni dans le concours d'agrégation des lycées : section indivise de sciences à l'École normale, agrégation unique de sciences. Mais bientôt la critique de cette organisation se manifeste, comme le montre, par exemple, la lettre d'un régent de mathématiques au journal Le Lycée ${ }^{22}$, en $1828:$ il suggère la séparation des sciences physiques et mathématiques à l'agrégation, et conteste principalement l'exigence de connaissances chimiques pour celui qui doit enseigner les mathématiques.

Une étape vers la spécialisation semble franchie en 1830 avec le nouveau règlement ${ }^{23}$ des études de l'École normale qui distingue deux divisions en $2^{e}$ et $3^{e}$ année de la section des sciences : la première pour les " sciences mathématiques et physiques " (ou division des « mathématiciens et physiciens "), la seconde pour les " sciences naturelles " (ou division des "chimistes et naturalistes"; en outre, la $1^{\text {re }}$ année d'études, commune aux deux sections, comporte des mathématiques, de la physique et de l'histoire naturelle (botanique). La définition de ces deux divisions rapproche chimie et histoire naturelle, tout en maintenant unies mathématiques et physique conformément à la ligne défendue par Poisson. En fait, les études de la première division incluent encore de la chimie et de l'histoire naturelle (minéralogie et géologie) alors que celles de la seconde division ne contiennent ni mathématiques ni physique et font une plus large part à l'histoire naturelle. Finalement, l'arrêté du 11 novembre 1831 rétablit une section des sciences indivise avec un plan d'études qui reproduit quasiment à l'identique celui de la première division prévue par le règlement de 1830 .

Or le règlement de 1830 associait à la définition des deux divisions la distinction de différents concours d'agrégation suivant les spécialités définies. Par son abandon, physique et chimie ne sont pas séparées, et on peut y voir un succès des chimistes dans le jeu des rivalités scientifiques. Le Journal général de l'Instruction publique note ${ }^{24}$ qu'au concours unique d'agrégation les sciences physiques et naturelles sont "comme absorbées dans la chimie et les mathématiques " ${ }^{25}$. Finalement, en octobre 1840 , le concours est scindé en deux spécialités, sciences mathéma-

22. Cf. t. IV, p. $177-178$.

23. Règlement du 30 oct. 1830, Bulletin universitaire, t. II, 1830-1831, p. 189.

24. 3 oct. 1840 , p. 576-577.

25. De 1834 à 1838 , période pendant laquelle J.-B. Dumas est au jury, la composition de sciences physiques est essentiellement une composition de chimie. 
tiques, d'une part, sciences physiques et naturelles, d'autre part. Dans ce choix, effectué après la mort de Poisson ${ }^{26}$, la physique est détachée des mathématiques et soumise à l'influence des chimistes bien que figurant comme une entité distincte de la chimie; désormais, trois compositions écrites sont prévues - physique, chimie, histoire naturelle - au lieu des deux compositions - physique ou chimie, mathématiques - de l'organisation antérieure. Ainsi une place est faite à l'histoire naturelle. En liaison avec cette spécialisation de l'agrégation, deux divisions sont alors créées en $3^{\mathrm{e}}$ année d'École normale.

La spécialisation était jugée nécessaire pour avoir des professeurs scientifiquement compétents, mais elle imposait un choix sur les couplages disciplinaires où la physique occupe une place charnière. On peut considérer que le choix retenu en 1840 a influé sur l'orientation de l'enseignement de cette discipline et, par conséquent, sur la formation des physiciens français. Nous avons déjà noté, à propos des doctorats, un éclatement de la physique en une physique expérimentale et une physique mathématique; nous soulignerons ici la conception de la physique du chimiste Jean-Baptiste Dumas qui, niant ainsi le rôle fécond de la théorie, affirme :

« la physique est une science expérimentale qui tire parti des mathématiques pour coordonner et exposer ses découvertes, et non point une science mathématique qui se soumettrait au contrôle de l'expérience $"{ }^{27}$.

\section{Les études secondaires}

Dans la première moitié du XIX ${ }^{\mathrm{e}}$ siècle est débattu le problème de la place accordée à l'enseignement scientifique dans un enseignement secondaire dominé par les humanités classiques; en particulier, pour obtenir le grade de bachelier ès sciences, il faut préalablement obtenir celui de bachelier ès lettres. Les discussions sont centrées autour de la question de la fusion ou de la séparation des lettres et des sciences. Si la classe où débute l'enseignement scientifique varie d'un plan d'études à

26. Après la disparition de Siméon Denis Poisson, Louis Poinsot est nommé au Conseil royal et devient le représentant des mathématiques, mais ses positions étaient différentes de celles de Poisson. Joseph BerTrand - dans son éloge historique de Louis Poinsot publié dans le Journal des savants, juillet 1872 , et cité in L. Ponssot, Éléments de statique, Paris, Gauthier-Villars, 1877 , p. IX-XXVIII - souligne que sur " aucune théorie mathématique » Poisson et Poinsot n'étaient "en communauté d'idées".

27. Jean-Baptiste Dumas, in Réferme de l'enseignement. Recueil des lois, décrets, instructions, circulaires et notes ministérielles pendant le ministère de H. Fortoul, t. II.1, 1854-1856, Paris, Delalain, 1856, p. 509. 
l'autre, la physique demeure en fin de cursus, en classe de philosophie. En 1826 (plan d'études du 16 septembre), le cours de sciences physiques qui comprend la chimie, la physique et l'histoire naturelle commence dès la première année de philosophie, avec un cours de physique élémentaire la première année et un cours de physique spéciale la seconde année. C'est dans cette dernière qu'après avoir décrit « les moyens de mesure et d'observation ", on présente des " développements sur les phénomènes " décrits successivement en première année ${ }^{28}$.

Après des oscillations dans les plans d'études en 1830, 1833, changeant la classe où débutent les mathématiques et l'histoire naturelle, l'ensemble de l'enseignement scientifique est refoulé en classe de philosophie, en 1840, sous le ministère de Victor Cousin qui justifie ainsi cette mesure : " La philosophie, les mathématiques, les sciences physiques et naturelles doivent venir après » les humanités car « elles s'adressent à la réflexion naissante ${ }^{29}$. Mais les inconvénients pédagogiques d'une telle organisation vont être dénoncés, et la nécessité de connaissances mathématiques pour aborder le cours de physique est nettement soulignée par les professeurs ${ }^{30}$. Le régime d'études instauré par Victor Cousin ne va pas durer; des débats ont lieu et ils aboutissent à la préparation de rapports par J.-B. Dumas et au statut de 1847 qui redéploie l'enseignement scientifique sur plusieurs classes.

J.-B. Dumas, dans des notes manuscrites ${ }^{31}$, insiste sur la nécessité d'un ordre logique dans l'enseignement des sciences ce qui implique de débuter par l'enseignement des mathématiques "qui n'emprunte rien aux autres sciences ». Mais, pour éviter de surcharger les élèves avec ce développement de l'enseignement scientifique, il propose de « réduire la géométrie aux propositions vraiment usuelles, l'algèbre à ce qu'il faut pour étudier les éléments de physique et de mécanique, les sciences naturelles à ce qu'exige la connaissance de l'hygiène ou de l'économie politique ». Les ambitions sont ainsi limitées à une conception utilitaire des diverses sciences, considérées dans leurs rapports les unes avec les autres.

Au souci évident de définir les domaines des différentes disciplines est associée la préoccupation de préciser les lignes de partage et les zones de recouvrement. J.-B. Dumas parle des "divisions artificielles de la

28. Programme du $1^{\text {er }}$ avr. 1828.

29. V. Cousin, op. cit. supra n. 21, p. 225.

30. N. Hulin-Jung, op. cit. supra n. 4, p. $57,59$.

31. Archives de l'Académie des sciences, cartons Dumas. 
science " et, au niveau de la formation des maitres, il manifeste une volonté de décloisonnement des disciplines en parfait accord avec sa conception de l'enseignement des sciences dans les lycées; il affirme ${ }^{32}$, en effet :

«L'enseignement classique de la science ne sera constitué que lorsqu'il se trouvera dans les collèges des professeurs [...] décidés à maintenir sa continuité par des soudures habiles, et des pénétrations fréquentes de l'une des divisions artificielles de la science dans l'autre."

Toutefois, la spécialisation des études s'impose, mais elle implique des choix pour le couplage des disciplines. La physique apparaît ainsi à un nœud des connaissances, éclatée en une physique expérimentale liée à la chimie - elle-même liée à la minéralogie - et une physique mathématique qui a place aux côtés des mathématiques. On a vu l'hésitation à coupler la physique aux mathématiques ou à la chimie pour l'agrégation des lycées. Par le choix effectué en 1840, l'enseignement de la physique est soumis à l'influence dominante des chimistes. Comme le notait Guillaume Libri ${ }^{33}$ en 1840 , la chimie l'emporte sur la physique par son intérêt économique :

"Si la chimie est la science que l'on cultive actuellement avec le plus d'ardeur et de succès [...] la physique ne se trouve pas dans un état aussi prospère [...]. En chimie organique le champ est vaste et permet de riches moissons. Malheureusement, la physique ne mène pas, comme la chimie à la fortune, et, dans notre siècle le moindre perfectionnement sur la fabrication du sucre de betterave aura toujours plus de retentissement dans le public que toutes les belles recherches de M. Savart sur l'acoustique, ou de M. Becquerel sur l'électricité. "

Cependant la nécessité des mathématiques est affirmée comme un instrument dans l'acquisition de la connaissance scientifique. Si on ne se contente pas d'un enseignement descriptif limité à des notions, il est nécessaire d'organiser l'enseignement dans un ordre logique, avec les mathématiques à la base.

Au fur et à mesure que les sciences progressent l'extension du domaine des connaissances impose la spécialisation des études, fondamentalement

32. Ibid.

33. Lettre à un américain sur l'état des sciences en France, Revue des Deux Mondes, 15 mars $1840,1^{\text {re }}$ lettre, p. 813 . 
liée à des choix de couplage disciplinaire, alors que dans le même temps les limites de séparation vont en s'estompant.

Nicole Hulin, Université Paris ИI et Centre Alexandre-Koyré, Muséum national d'histoire naturelle, Pavillon Chevreul, 57, rue Cuvier, 75231 Paris Cedex 05

(avril 1992). 